Supporting Information

for

\title{
Single-Molecule Junction Formation in Break-Junction Measurements
}

\author{
Tianren $\mathrm{Fu}^{1 \ddagger}$, Kathleen Frommer ${ }^{1^{\ddagger}}$, Colin Nuckolls ${ }^{1}$, Latha Venkataraman*1,2 \\ ${ }^{1}$ Department of Chemistry, Columbia University, New York, New York 10027, United States \\ ${ }^{2}$ Department of Applied Physics and Applied Mathematics, Columbia University, New York, New \\ York 10027, United States
}

$\$$ These authors contributed equally.

Email:1v2117@columbia.edu

\section{Contents:}

1. Additional Data

2. Snapback Measurement Details

3. Details of the Neural Network Method

4. References 


\section{Additional Data}

\section{Sample trace from a measurement with a molecule}

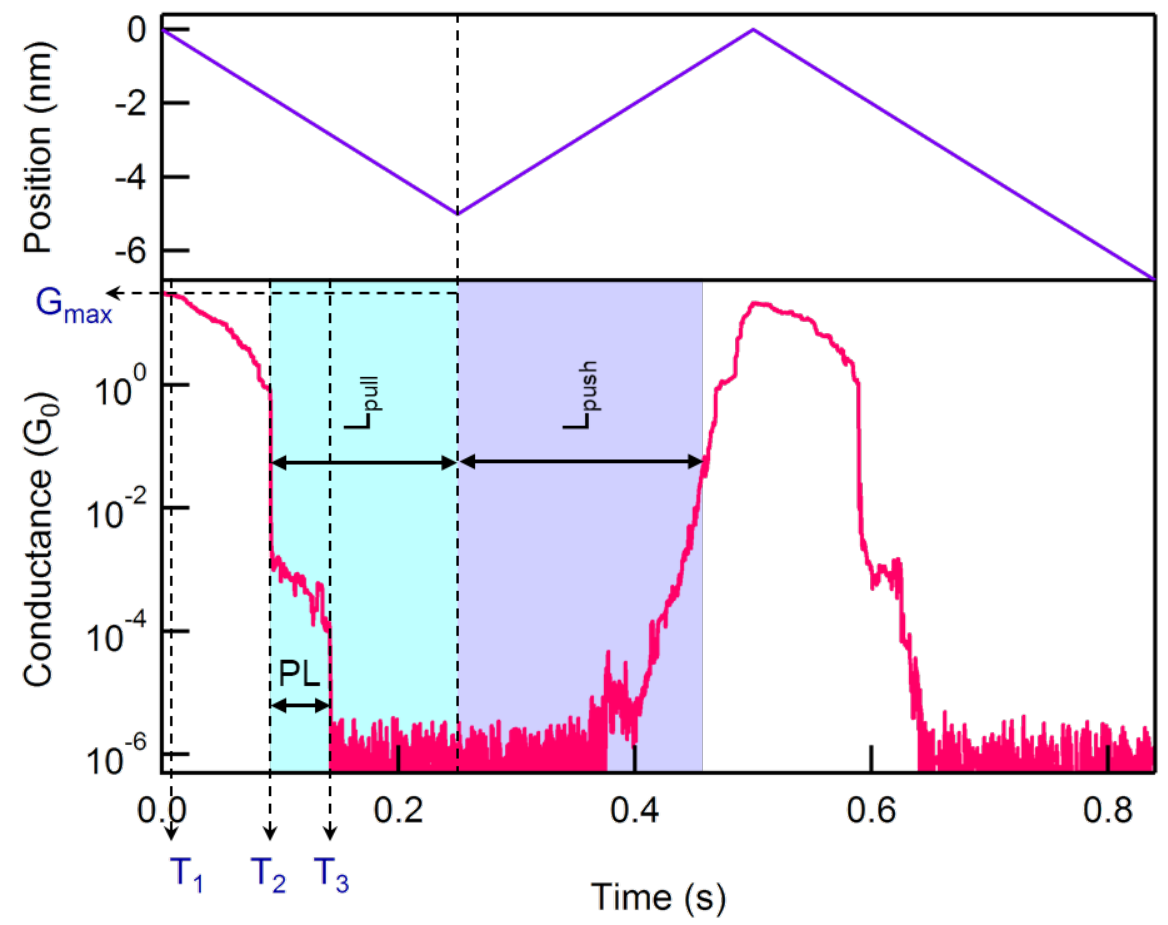

Figure S1. Sample piezo ramp (upper panel) and conductance versus time trace (lower panel) for a push-pull trace measured in a $0.1 \mathrm{mM} 1,2,4$-trichlorobenzene (TCB) solution of 4,4"-diamino- $p$ terphenyl at an applied bias of $100 \mathrm{mV}$ and pulling (and pushing) rate of $15 \mathrm{~nm} / \mathrm{s}$. $\mathrm{T}_{1}$ indicates the time when the highest conductance $\left(\mathrm{G}_{\max }\right)$ is observed; $\mathrm{T}_{2}$ is the time when the initial Au contact ruptures; $\mathrm{T}_{3}$ is the time when the Au-molecule-Au junction breaks. $\mathrm{L}_{\text {pull }}$ indicates the distance pulled after the contact breaks and $\mathrm{L}_{\text {push }}$ indicates the distance pushed before the contact is reformed. PL is the length of molecular junction plateau. For an experiment with a solution of the target molecules, the snapback is determined in the same way as in a measurement with solvent alone, i.e., snapback $=L_{\text {push }}-L_{\text {pull, }}$, and the molecular plateau length is defined as the distance between the first and last point of the conductance trace that falls in the molecular conductance region. We only consider traces that have a molecular plateau if the plateau is longer than $0.01 \mathrm{~nm}$. 

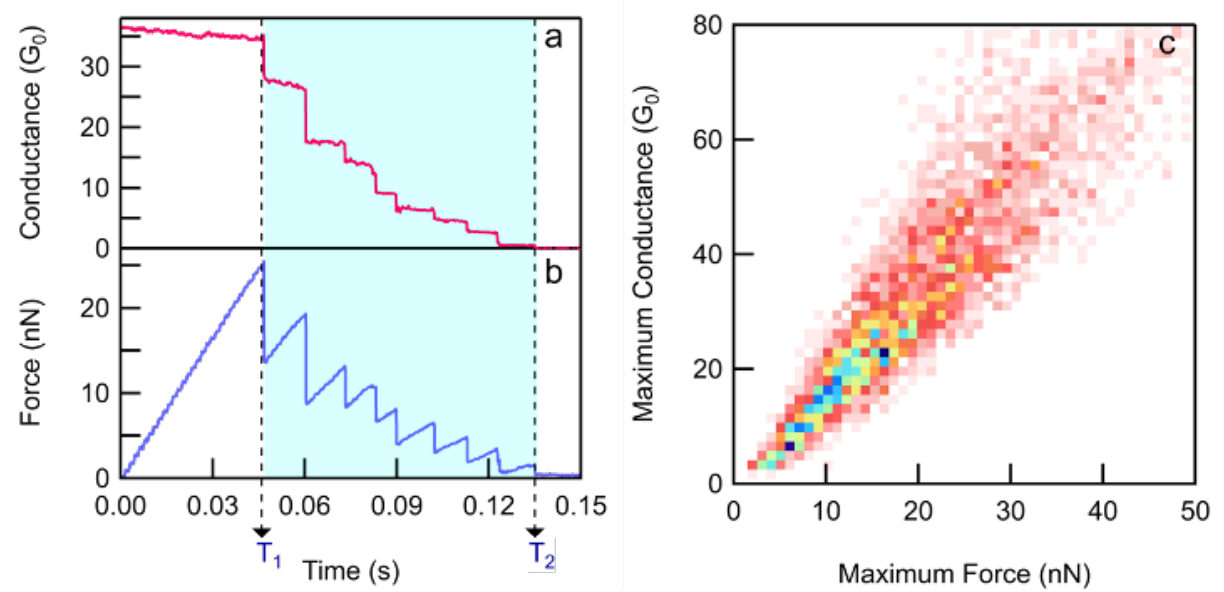

Figure S2. (a) Sample conductance versus time trace and (b) force versus time trace for a clean Au substrate measured with a pull rate of $18 \mathrm{~nm} / \mathrm{s}$ and applied bias of $50 \mathrm{mV}$ using a gold-coated AFM cantilever as the tip and a standard Au-coated substrate. $T_{1}$ is the time when the highest force is reached and the first rearrangement event occurs. $\mathrm{T}_{2}$ is the time when the Au-Au contact ruptures. The light blue shaded region is the time over which the Au contact is thinning. (c) 2D correlation histogram of $\mathrm{G}_{1}$ vs maximum force constructed from 3966 measured force and conductance traces.

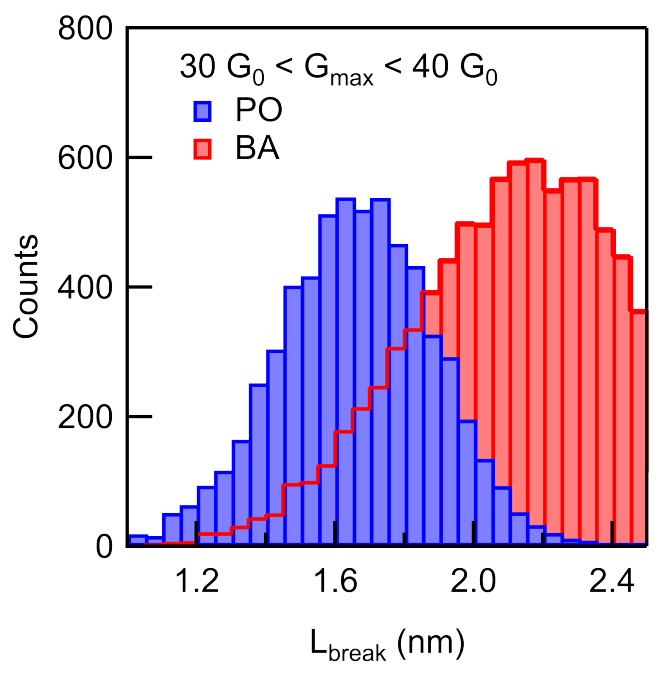

Figure S3. $\mathrm{L}_{\text {break }}$ histograms for measurements in PO and BA solvents without molecules where we have selected a subset of traces that have a $G_{\max }$ between 30 and $40 \mathrm{G}_{0}$. We see that $\mathrm{L}_{\text {break }}$ is larger in BA than PO in agreement with the data shown in Figure 4. 

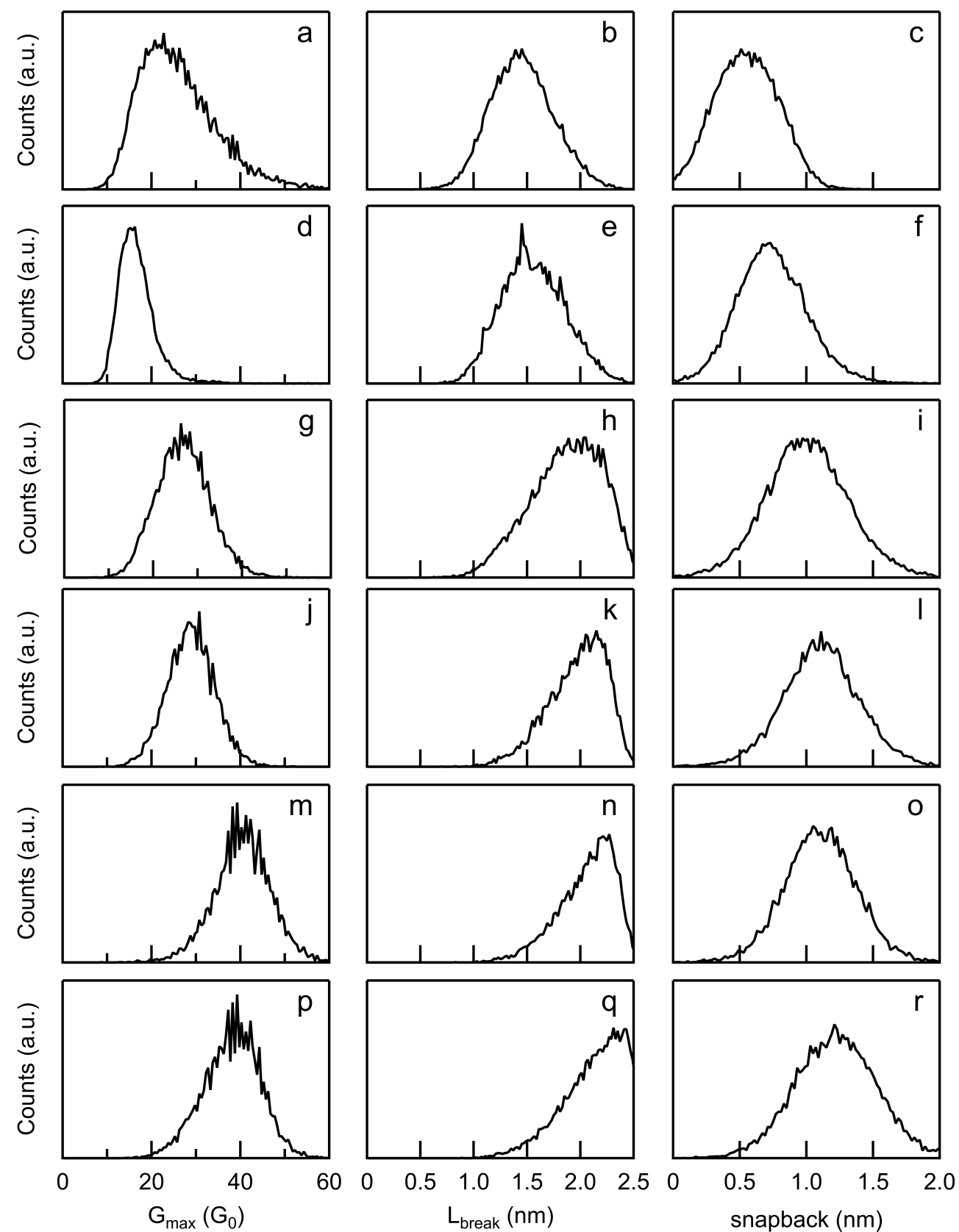

Figure S4. Distribution histograms of parameters for pure solvent experiments. From top to bottom: different solvents, (a-c) 1-phenyloctane (PO), (d-f) tetradecane (TD), (g-i) 1,2,4-trichlorobenzene (TCB), (j-l) 1-bromonaphthalene (BN), (m-o) 1-iodonaphthalene (IN) and (p-r) 4-bromoanisole (BA). 

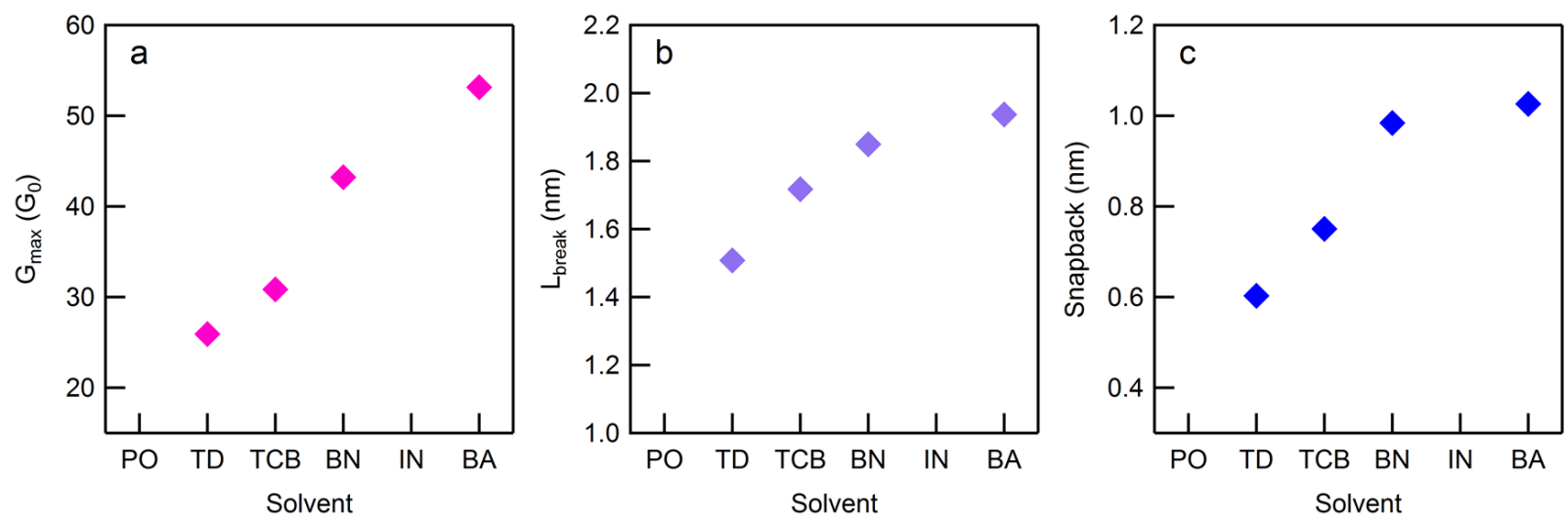

Figure S5. (a) The most probable $\mathrm{G}_{\max }$, (b) $\mathrm{L}_{\text {break, }}$ and (c) snapback for $0.1 \mathrm{mM} 4,4$ "-diamino- $p$ terphenyl solutions measured in four different solvents. Abbreviations for solvents used are as follows: $\mathrm{TD}=$ tetradecane, $\mathrm{TCB}=1,2,4$-trichlorobenzene, $\mathrm{BN}=1$-bromonaphthalene, $\mathrm{BA}=4$-bromoanisole.
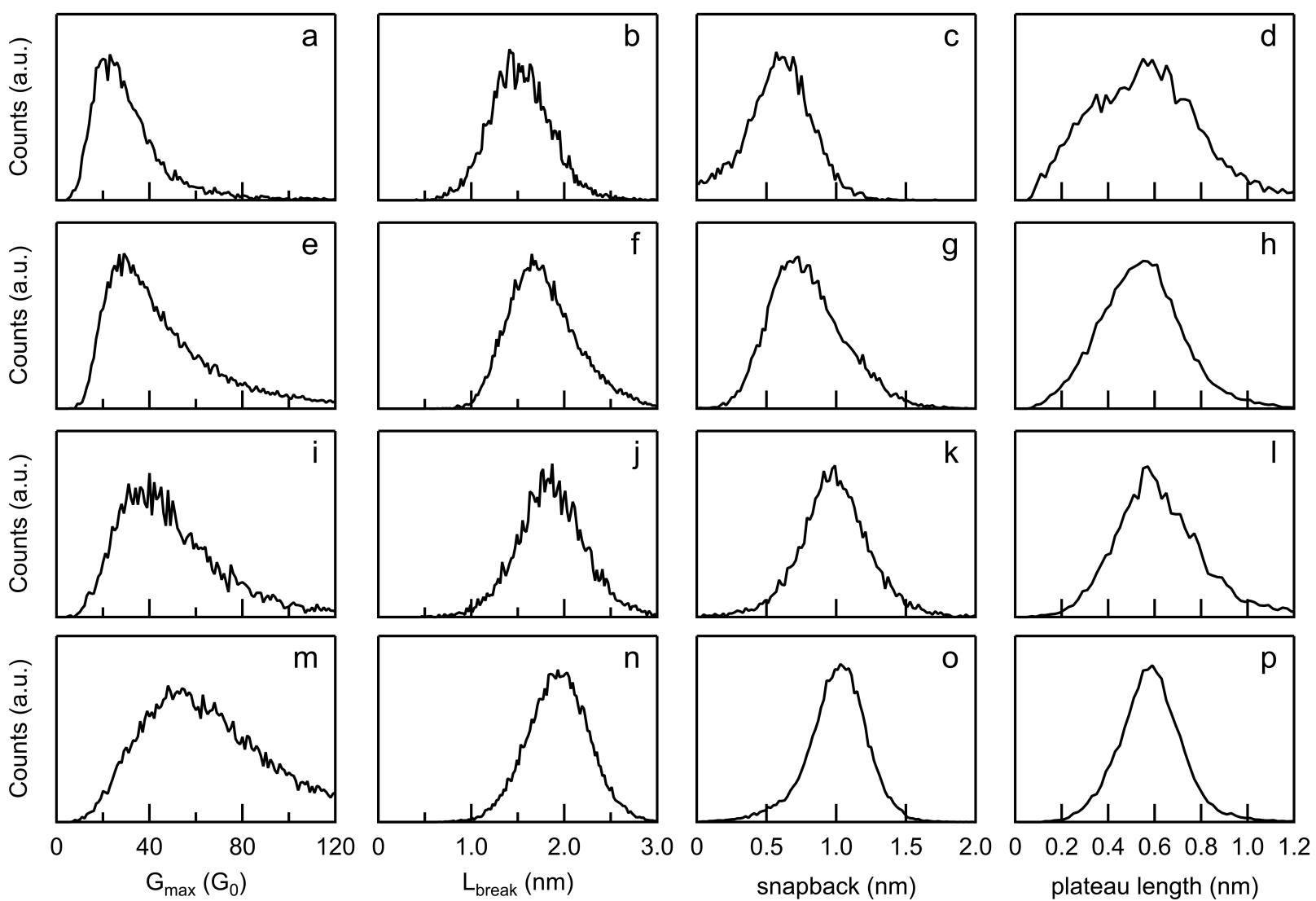

Figure S6. Distribution histograms of parameters for experiments of $0.1 \mathrm{mM} 4,4$ "-diamino- $p$ terphenyl solutions. From top to bottom: different solvents, (a-d) tetradecane (TD), (e-h) 1,2,4trichlorobenzene (TCB), (i-l) 1-bromonaphthalene (BN) and (m-p) 4-bromoanisole (BA). 

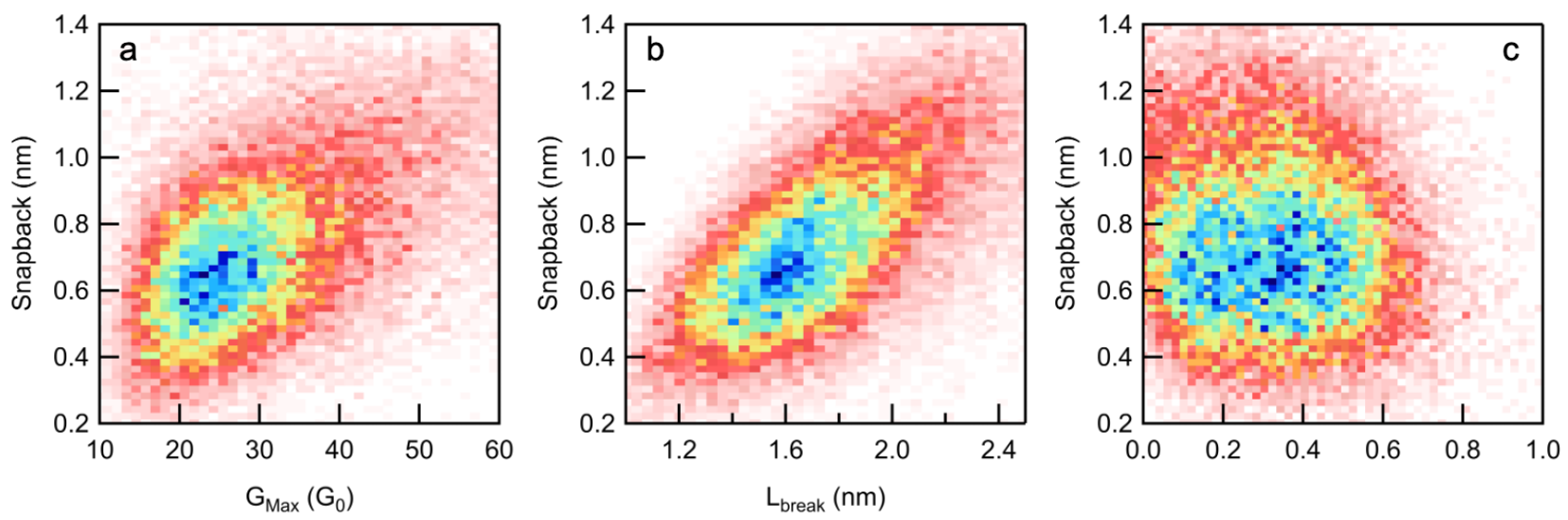

Figure S7. Two-dimensional (2D) correlation histograms constructed from 27940 selected push-pull traces of a $0.1 \mathrm{mM}$ solution of 1,3-propanediamine in TCB. Snapback versus (a) $\mathrm{G}_{\max }$ with a Pearson correlation coefficient of 0.443 ; (b) Lbreak with a Pearson correlation coefficient is 0.683 and (c) Molecular plateau length with a Pearson correlation coefficient is -0.118 . 


\section{Snapback Experiment Details}

As explained in the main text, we design the modified push-pull STM-BJ experiment to measure the snapback distance of the Au electrodes after the junction ruptures. This distance is $\mathrm{L}_{\text {push }}-\mathrm{L}_{\text {pull }}$ from these push-pull measurements and determines the relaxation over a millisecond timescale. Figure S5a shows a simplified illustration of this experiment in a solvent, and compliments Figure 1.
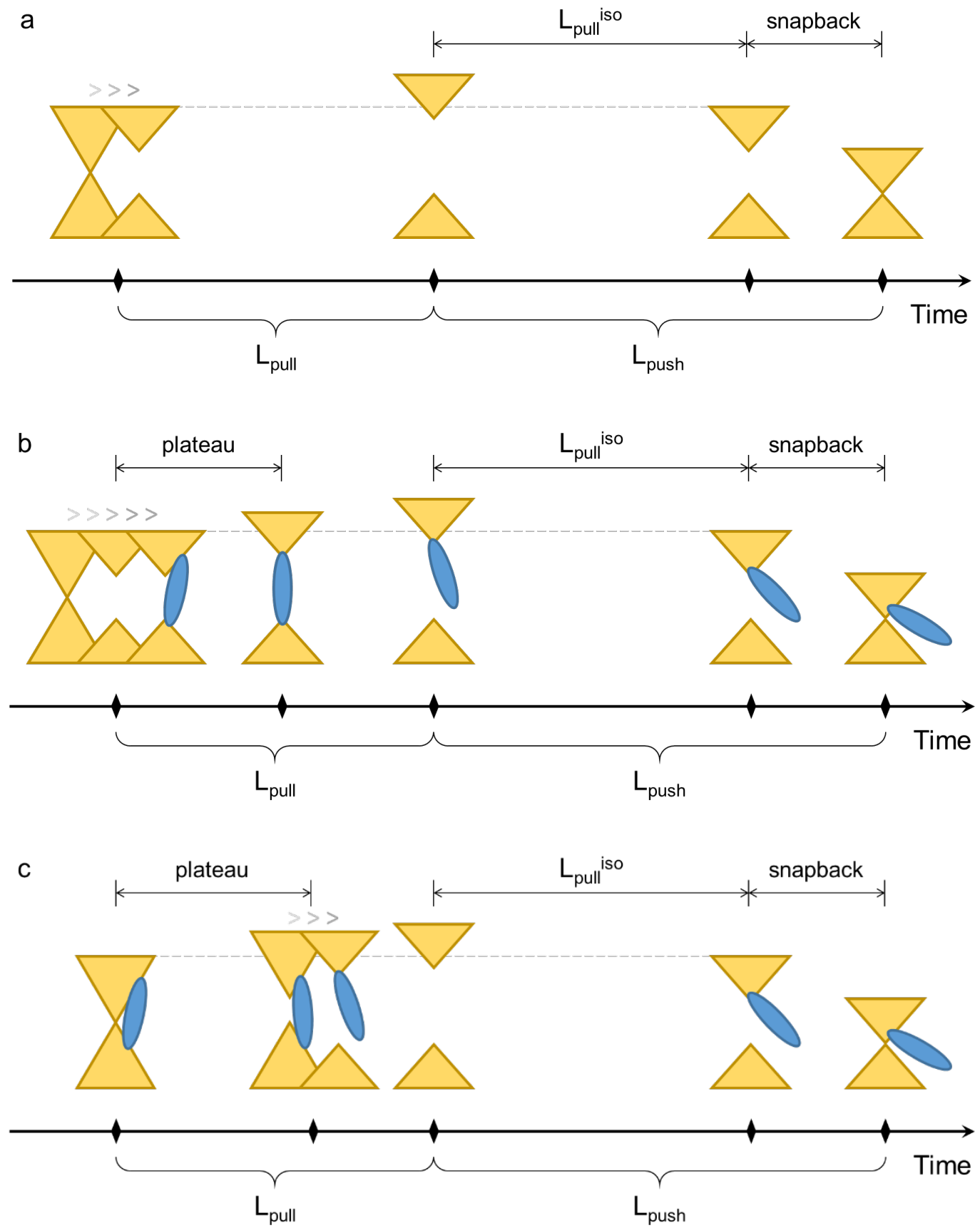

Figure S5. Illustration of a push-pull STM-BJ experiment in (a) on pure solvent, (b) solvent with a target molecule where the molecule is captured after the rupture of $\mathrm{Au}$-Au contact, and (c) solvent with with a target molecule where the molecule bridges the Au electrodes in parallel to the point-contact. The yellow triangles represent Au electrodes, and the blue ovals represent the molecules. 
Going from left to right, Figure S5a starts with an Au-Au contact that ruptures then snapbacks back. After the contacts are pulled apart by a distance $\mathrm{L}_{\text {pull, }}$ we start to push the electrodes towards each other until a contact is formed. The distance the electrodes are pushed together is denoted as $\mathrm{L}_{\text {push }}$. $\mathrm{L}_{\text {push }}$ includes the distance that the electrodes are withdrawn (denoted as $\mathrm{L}_{\text {pull }}{ }^{\text {iso }}$ ) and the amount that the electrodes relax, which is the snapback. Note that most of the relaxation occurs as soon as the contacts rupture, while some slower process that reorganize the electrodes also lead to an enlargement of the gap between the electrodes. ${ }^{1}$.

For experiments with molecules, the snapback is measured in the same way. In both cases, the threshold for $\mathrm{Au}-\mathrm{Au}$ contact is chosen to be $0.05 \mathrm{G}_{0}$ which is higher than the molecular conductance of all the molecules studied in this work. Hence, the snapback measurement does not interfere with the molecular junction plateau. This process is illustrated in Figure S5b and S5c, where we hypothesize that the snapback happens at the same stage as in the pure solvent experiments.

Figure S5b illustrates a path where the molecular junction is formed after the contacts rupture. Here, the snapback occurs immediately after the Au-Au point contact ruptures as in the case of the pure solvent experiments illustrated in Figure S5a. A molecule is captured into this gap after the electrodes relax.

Figure S5c shows a junction where a molecule is already bound bridging the electrodes in parallel to the $\mathrm{Au}$ point-contact, the scenario that we argue here dominates in the measurements. Since the molecule is already present when the point contact breaks, the extent of the snapback is very much decreased since the two electrodes are still held together by the molecule, and thus under some tension. The electrodes relax fully only after the Au-molecule-Au junction finally breaks. We can still measure this snap-back as we do in the case without molecules, as $\mathrm{L}_{\text {push }}-\mathrm{L}_{\text {pull. }}$.

Irrespective of whether a molecule is present before or after the electrodes relax, the maximum extension of a Au-molecule-Au should be a stochastic value with a molecule-dependent constant mean. For the path detailed in Figure S5b, the molecular plateau length should be this maximum extension minus the snapback, because the molecular junction starts with an initial displacement of the snapback. This would result in a strong negative correlation between the plateau length and the snapback, which is not observed in our experiments. For the path shown in Figure S5c however, since most of the snapback takes place after the molecular junction breaks, the measured plateau length is still bound by the maximal molecule-dependent value but depends on the details of the junction configuration, not on the snapback.

To rationalize the small negative correlation seen in the experiments between the molecular plateau length and the snapback, this likely indicates the electrodes could relax partially after the Au$\mathrm{Au}$ contact is ruptured. This explanation is reasonable because the strength of the $\mathrm{Au}-\mathrm{Au}$ bond is greater than that of the linker-Au bond. ${ }^{2}$ 


\section{Details of the Neural Network Method}

We build two convolutional neural network $(\mathrm{CNN})$-based models that predict snapback and molecular plateau length from the Au conductance region of an STM-BJ conductance trace. Based on the performance of these two models, we can learn the connection between the snapback or plateau length and the Au contact conductance evolution with length. ${ }^{3-5}$ Using this method, we eliminate the errors that could be there if instead of using the entire trace, we simply model the relations using a few key parameters. The drawback of this $\mathrm{CNN}$ structure is that it is hard to figure out which characteristics of the traces are important features in determining snapback or plateau lengths.

In these two models, we use as input, the Au conductance region of the raw STM-BJ conductance trace in its initial pulling phase. The other parts of the trace are cut off and set to zero to prevent the model from directly reading out the snapback from the complete trace. Our model consists of 3 convolutional layers followed by a global average pooling and then a fully-connected layer. Each of these convolutional layers is a convolutional-batch normalization ${ }^{6}$-dropout ${ }^{7}$ structure, where for the convolutional part, the kernel size $=21$ points, the stride $=6$ points, and the number of channels is 32 . The width of the fully-connected layer is 8 . Activation using rectified linear units (ReLU) are applied after each of these layers.

We study the dataset of 24,880 traces from the experiment in a 1,2,4-trichlorobenzen (TCB) solution of 4,4 "-diamino- $p$-terphenyl. We use $90 \%$ of these traces to train the models and the other $10 \%$ for model validation. Training of these models was fulfilled by TensorFlow ${ }^{8}$.
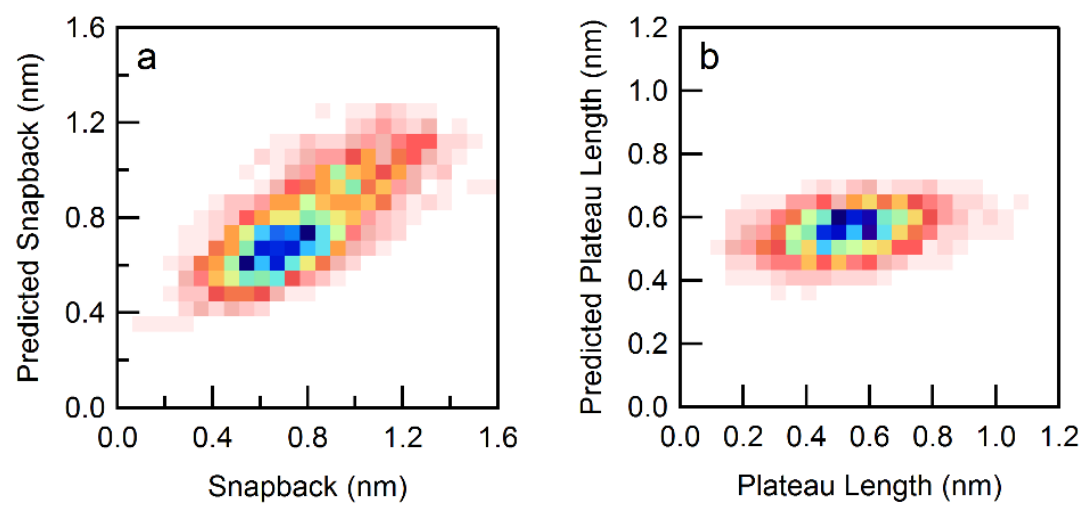

Figure S6. The 2D dimensional correlation histograms between the measured and the model-predicted values for (a) snapback and (b) molecular plateau length, according to the CNN-based models.

Using the validation dataset, which is not used during the training process, we obtain a correlation of $73.1 \%$ for snapback and $32.4 \%$ for the plateau length (Figure S6). The magnitude of this correlation can indicate how much the Au contact evolution history can determine the snapback or plateau length. We see that the snapback is determined by the evolution of the Au contact while the plateau length is not. 


\section{References}

1. Kim, T.; Vazquez, H.; Hybertsen, M. S.; Venkataraman, L., Conductance of molecular junctions formed with silver electrodes. Nano Lett 2013, 13 (7), 3358-64.

2. Frei, M.; Aradhya, S. V.; Koentopp, M.; Hybertsen, M. S.; Venkataraman, L., Mechanics and chemistry: single molecule bond rupture forces correlate with molecular backbone structure. Nano Lett 2011, 11 (4), 1518-23.

3. Fu, T.; Zang, Y.; Zou, Q.; Nuckolls, C.; Venkataraman, L., Using Deep Learning to Identify Molecular Junction Characteristics. Nano Lett 2020, 20 (5), 3320-3325.

4. Huang, F.; Li, R.; Wang, G.; Zheng, J.; Tang, Y.; Liu, J.; Yang, Y.; Yao, Y.; Shi, J.; Hong, W., Automatic classification of single-molecule charge transport data with an unsupervised machinelearning algorithm. Phys Chem Chem Phys 2020, 22 (3), 1674-1681.

5. Lauritzen, K. P.; Magyarkuti, A.; Balogh, Z.; Halbritter, A.; Solomon, G. C., Classification of conductance traces with recurrent neural networks. J Chem Phys 2018, 148 (8), 084111.

6. Ioffe, S.; Szegedy, C. Batch Normalization: Accelerating Deep Network Training by Reducing Internal Covariate Shift arXiv e-prints [Online], 2015. https://ui.adsabs.harvard.edu/abs/2015arXiv150203167I (accessed February 01, 2015).

7. Srivastava, N.; Hinton, G.; Krizhevsky, A.; Sutskever, I.; Salakhutdinov, R., Dropout: a simple way to prevent neural networks from overfitting. J. Mach. Learn. Res. 2014, 15 (1), 1929-1958.

8. Abadi, M. n.; Agarwal, A.; Barham, P.; Brevdo, E.; Chen, Z.; Citro, C.; Corrado, G. S.; Davis, A.; Dean, J.; Devin, M.; Ghemawat, S.; Goodfellow, I.; Harp, A.; Irving, G.; Isard, M.; Jia, Y.; Jozefowicz, R.; Kaiser, L.; Kudlur, M.; Levenberg, J.; Mane, D.; Monga, R.; Moore, S.; Murray, D.; Olah, C.; Schuster, M.; Shlens, J.; Steiner, B.; Sutskever, I.; Talwar, K.; Tucker, P.; Vanhoucke, V.; Vasudevan, V.; Viegas, F.; Vinyals, O.; Warden, P.; Wattenberg, M.; Wicke, M.; Yu, Y.; Zheng, X. TensorFlow: Large-Scale Machine Learning on Heterogeneous Distributed Systems arXiv e-prints [Online], 2016. https://ui.adsabs.harvard.edu/abs/2016arXiv160304467A (accessed March 01, 2016). 